\title{
Siloed Federated Learning for Multi-Centric Histopathology Datasets
}

\author{
Mathieu Andreux ${ }^{1}$, Jean Ogier du Terrail ${ }^{1}$, \\ Constance Beguier, and Eric W. Tramel \\ Owkin, Inc., New York, NY, USA \\ firstname.lastname@owkin.com
}

\begin{abstract}
While federated learning is a promising approach for training deep learning models over distributed sensitive datasets, it presents new challenges for machine learning, especially when applied in the medical domain where multi-centric data heterogeneity is common. Building on previous domain adaptation works, this paper proposes a novel federated learning approach for deep learning architectures via the introduction of local-statistic batch normalization (BN) layers, resulting in collaboratively-trained, yet center-specific models. This strategy improves robustness to data heterogeneity while also reducing the potential for information leaks by not sharing the center-specific layer activation statistics. We benchmark the proposed method on the classification of tumorous histopathology image patches extracted from the Camelyon 16 and Camelyon17 datasets. We show that our approach compares favorably to previous state-of-the-art methods, especially for transfer learning across datasets.
\end{abstract}

Keywords: Federated Learning $\cdot$ Histopathology $\cdot$ Heterogeneity $\cdot$ Deep Learning

\section{Introduction}

Federated learning (FL) has recently emerged as a new paradigm for scalable and practical privacy-preserving machine learning (ML) on decentralized datasets 22 . In the case of medical data, notably digital histopathology images, this approach brings the promise of ML architectures trained over large and diverse populations, a necessary component for truly generalizable medical findings. By bridging the gap between localized, curated, and non-portable per-institution datasets, a federated approach permits the study of otherwise unconstructible research datasets. With the ability to utilize large and rich datasets for ML, medical researchers have the potential to make new scientific discoveries, as evidenced in [8].

Two challenges currently limit the applicability of existing FL techniques to real-world histopathology datasets: inter-center data heterogeneity and wellunderstood privacy assurances on communicated model parameters. Statistical

\footnotetext{
${ }^{1}$ Contributed equally to this work.
} 
heterogeneity in the data distribution between participating centers is a key issue for FL 24]14 18, which may lead to model biases or even prevent training convergence [24]. Such heterogeneity is often present in histopathology datasets, where variations in staining procedure, scanning device configuration, and systematic imaging artifacts are commonplace [16].

In this paper, we propose a novel federated learning strategy, called SiloBN, which brings improvements both in terms of resilience to heterogeneity and privacy. This strategy relies on batch normalization $(\mathrm{BN})$ 13 layers, which are ubiquitous in deep learning (DL), especially for computer vision applications. SiloBN introduces, or uses already specified, local-statistic BN layers within DL architectures. More precisely, we propose the following novel contributions:

i) We demonstrate the applicability of FL to real-world tile-level digital pathology image classification, using the Camelyon16 and Camelyon17 datasets 21] (Sec. 5). To the best of our knowledge, this work is among the first examples thereof. Previous works rather relied on the SplitLearning framework [30].

ii) We introduce SiloBN, a new FL approach for training DL models robust to inter-center data variability by introducing local-statistic BN layers. This approach also reveals less sensitive information by not communicating local activation statistics (Sec. 4).

iii) We show that our proposed approach achieves same or better performance than existing federated techniques for intra-center generalization (Sec. 5.2), even in challenging settings, and yields better out-of-domain generalization results (Sec. 5.3).

\section{Background}

In the years since the publication of the 2013 ICPR-winning approach of [7, the application of DL to digital pathology tasks has blossomed 9 930. In parallel, federated learning [26 22], a distributed privacy-preserving ML paradigm, has recently experienced incredible growth as a topic of study, garnering much interest in the medical research community. Due to the highly sensitive nature of the medical data, techniques such as FL are a requirement for investigators in order to develop state-of-the-art ML models over a fractured and highly regulated data landscape.

Federated Learning As presented in 22, the aim of FL is to obtain a single well-trained model from a distributed network of $K$ participants, each possessing their own privately-held datasets. Generically, this is accomplished by optimizing model parameters $\theta$ w.r.t. an expectation over the individual participant losses $\ell_{i}$, i.e. $\mathcal{L}(\theta) \triangleq \mathbb{E}_{i}\left[\ell_{i}(\theta)\right]$. This optimization is carried out iteratively in federated rounds, where at each round $t$ the expectation is approximated as $\sum_{i \in \mathcal{C}^{(t)}} \alpha_{i} \ell_{i}(\theta)$, where $\mathcal{C}^{(t)} \sim[K]$ is random sub-sampling of participants and $\alpha_{i} \geq 0$ are contribution weightings. The authors propose federated averaging (FedAvg) to reduce coordination rounds by performing several local optimization 
steps prior to aggregation,

$$
\overbrace{\theta_{i}^{(t)}=\mathrm{Opt}_{E}\left(\theta^{(t)}, \ell_{i}\right)}^{\text {Local }}, \quad \overbrace{\theta^{(t+1)}=\sum_{i \in \mathcal{C}^{(t)}} \frac{N_{i}}{N^{(t)}} \theta_{i}^{(t)}}^{\text {Central Server, }}
$$

where $N_{i}$ is the number of data samples at participant $i$, and $N^{(t)} \triangleq \sum_{i \in \mathcal{C}^{(t)}} N_{i}$, $\mathrm{Opt}_{E}(\cdot)$ is an $E$-step iterative optimization, and $t$ indexes federated rounds. For brevity, we refer the reader to the original work of 22] for details. Further adaptations can be made to the algorithm to enhance privacy, e.g. [1], but are out of scope of the present work.

Data Heterogeneity in FL Data heterogeneity has been identified as a key open challenge for FL [18. For example, despite its practical success, FedAvg does not provide a natural guarantee of convergence, especially for highly dissimilar participant datasets. The FedProx algorithm of [24, similar to the EASGD of [31, introduces a quadratic loss term at each round $\ell_{i}^{(t)}\left(\theta_{i}\right)=\ell_{i}\left(\theta_{i}\right)+\frac{\lambda}{2}\left\|\theta_{i}-\theta^{(t)}\right\|^{2}$, to restrain local models from diverging during local optimization. This approach was shown to be effective on the large- $K$, heterogeneous LEAF datasets 6]. Novel participant sampling strategies have also been recently proposed [1910 to help adapt FedAvg to heterogeneous participant datasets. Finally, one can also seek to partition the set of participants into clusters of effective collaborators, as in [25]. It should be noted that the assumption that data samples are independent and identically distributed (i.i.d.) is core to many ML techniques, and empirical risk minimization [29] in particular. For FL, we retain an i.i.d. assumption on data intra-participant, but not inter-participant.

\section{FL in Healthcare}

The original application of FL sought to define techniques for distributed training over mobile edge devices, e.g. smart phones [17/22, and has now been practically scaled to $K>10^{6}$ [5]. Notably, this application also assumes that each participant has a paucity of training data, i.e. $N_{i} \ll N=\sum_{j=1}^{K} N_{j} \quad \forall i \in[K]$. The application of FL to the context of distributed and private medical datasets reverses the order of magnitude on both of these variables, with the number of participants often being quite low, e.g. $K \leq 10$, and generally having access to much larger and higher quality datasets at each center, i.e. $\log N_{i} \approx \log N$. The implication of this reversal is that the nature and goals of the federated system shift between the two settings:

i) Consumer FL - Federated training coordinated and of value to central service provider. No single participant, out of very many, can train an effective model, and no participant has an incentive to use such a local model. Goal of the service provider is to coordinate and distribute final global model back to participants. Service provider may hold their own private or public evaluation data. 
ii) Collaborative $F L$ - Federated training coordinated by central service provider on behalf of few participants, who value and seek to reuse its outputs. Participants have enough local data of quality to produce effective local models, but seek to augment this local task performance through collaboration. Evaluation data is sensitive and held by participants.

For this work, we consider the collaborative FL setting for training a tile-level classifier for WSI across multiple centers. This setting poses several challenges for the construction of distributed algorithms which are not fully addressed in the literature described in Sec. 2 .

Data heterogeneity is a key challenge for collaborative FL, as inconsistencies in data preparation will not be averaged out over a large population of participants. Additionally, in the consumer FL setting, $|\mathcal{C}(t)| \ll K$, which means outlier participants are rarely revisited. For collaborative FL, such strong sub-sampling is not desirable, as per-round updates would become more biased and also lead to potential privacy challenges as the sensitivity of the aggregated model w.r.t. the center of origin increases. For this reason, we consider only full-participation federated rounds in the case of collaborative FL. In order to keep stochasticity in the optimization, contrary to 22 which uses local epochs, we use $E$ to denote the number of local mini-batch updates in (1).

\section{Proposed Method}

Batch Normalization (BN) BN [13] is a critical component of modern deep learning architectures. A BN layer renormalizes an input tensor $x$ as

$$
\mathrm{BN}(x)=\gamma \frac{x-\mu}{\sqrt{\sigma^{2}+\epsilon}}+\beta,
$$

where $\mu$ and $\sigma^{2}$, hereafter denoted as BN statistics, are calculated as the running means and variances, respectively, of each channel computed across both spatial and batch dimensions, and $\gamma$ and $\beta$ are learned affine renormalization parameters, and where all computations are performed along the channel axis. BN has been used to both speed model training and also enhance model predictive performance [13].

While BN layers are common architectural components [11|12|28, they have not been thoroughly addressed in the federated setting, and are often simply ignored or removed [22]. Indeed, the naïve application of FedAvg would ignore the different roles of the local activation statistics $\left(\mu, \sigma^{2}\right)$, and the trained renormalization $(\gamma, \beta)$ and simply aggregate both at each federated round, as depicted in Fig. 1 (left). In the following, we use this method as a baseline when using $F e d A v g$ on a network with BN layers.

BN layers can also be used to separate local and domain-invariant information. Specifically, the BN statistics and learned BN parameters play different roles [20]: while the former encode local domain information, the latter can be transferred across domains. This opens interesting possibilities to tune models to local datasets, which we discuss in the following section. 


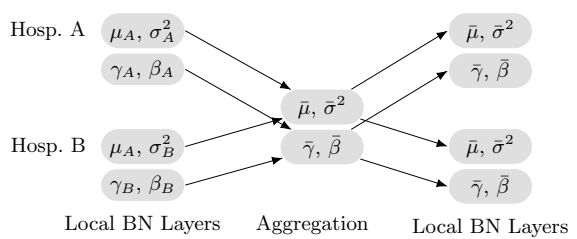

Naïve FedAvg Training

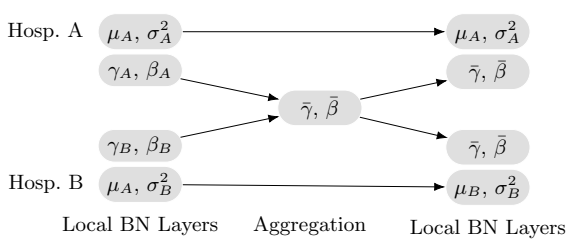

SiloBN Training

Fig. 1. Description of the different approaches to multi-center training of BN layers for two hospitals. In this description, all variables follow the definitions given in (2). Computation flows from left to right. Non-BN layers are shared in both methods.

SiloBN Instead of treating all BN parameters equally, we propose to take into account the separate roles of BN statistics $\left(\mu, \sigma^{2}\right)$ and learned parameters $(\gamma, \beta)$. Our method, called SiloBN, consists in only sharing the learned BN parameters across different centers, while BN statistics remain local. Parameters of non-BN layers are shared in the standard fashion. This method is depicted in Fig. 1 (right). Keeping BN statistics local permits the federated training of a model robust to the heterogeneity of the different centers, as the local statistics ensure that the intermediate activations are centered to a similar value across centers. While SiloBN can be applied to models which already possess BN layers, we demonstrate that in some cases, BN layers can be added to the base model to improve resilience to heterogeneity as well as domain generalization.

Model Personalization and Transfer A consequence of the proposed method is that one model per center is learned instead of a single global model, thereby enabling model personalization. However, it is not straightforward to generalize the resulting models to unseen centers, as BN statistics must be tuned to the target dataset. A simple approach to overcome this issue is to follow the $A d a B N$ [20] method, and recompute BN statistics on a data batch of the new target domain, while all other model parameters remain frozen to those resulting from the federated training.

Privacy and $B N$ When applying standard FL methods on a network containing $\mathrm{BN}$ layers, the $\mathrm{BN}$ statistics are shared between centers and may leak sensitive information from local training datasets. With SiloBN, the BN statistics are not shared among centers, and in particular the central server never has access to all the network parameters. As a number of privacy attacks $27 / 32$ are most effective with white-box, full-parameter, knowledge, the reduction of the amount of shared information can only help diminish the effectiveness of such attacks when compared to synchronizing batch activation statistics across participants. 


\section{Experiments}

\subsection{Datasets}

In order to understand the performance of federated algorithms in a practical setting, we seek real-world datasets with actual multi-centric origins. For histopathology, the Camelyon 16 [3] and Camelyon 17 [2] challenge datasets provide H\&E stained whole slide images (WSI) of lymph node sections drawn from breast cancer patients from 2 and 5 hospitals, respectively. Fig. 4 in Supp. Mat. shows representative slides from each center, which have different color statistics on average.

Building on Camelyon 16 and Camelyon 17, we construct two tile-level tumor classification histopathology datasets, referred to as FL-C16 and FL-C17. In both cases, the task consists in classifying the tiles between tumorous and healthy ones. In order to get tile-level annotations, tumorous tiles are extracted from pixel-level annotated tumorous WSI, whenever available, while healthy tiles are extracted from healthy WSI. This restriction, motivated by incomplete expert annotations, ensures tile-level labels' correctness. For each WSI, we extract at most 10, 000 non-overlapping fixed-size matter-bearing tiles uniformly at random using a U-Net segmentation model [23] trained for matter detection. To reduce class imbalance, for each healthy WSI, we cap the number of extracted tumornegative tiles to 1,000 for FL-C16 and 100 for FL-C17, as FL-C17 has fewer annotated tumor-positive tiles than FL-C16. Finally, each dataset is partitioned into a training set $(60 \%)$, a validation set $(20 \%)$, and a test set $(20 \%)$ using perhospital stratification. For FL-C16 (resp. FL-C17), tiles from same slide (resp. tiles from same patient) are put into the same partition. Table 3 in Supp. Mat. details the distribution of tiles in the data partitions.

\subsection{Experiments on FL Histopathology Datasets}

Baselines We compare the proposed SiloBN method to two standard Federated algorithms, FedAvg and FedProx, which treat BN statistics as standard parameters. Additionally, we report results obtained in the pooled setting, where the training sets of each center are concatenated and the origin center information is discarded during training. To measure the benefits of the personalization of our model, we also compare the proposed method to a local training, where on each center one model is trained independently without inter-center collaboration.

Training setting For tile classification, we use two deep convolutional neural network (DCNN) architectures that only differ by the presence or absence of BN layers, which we denote as DCNN and DCNN+BN, respectively. These architectures are detailed in Fig. 3 in Supp. Mat. Note that, due to computational constraints, this architecture is shallower than standard ones [11]228], yielding better performance without BN layers, which is not entirely realistic. Each FL algorithm is run with $E=1$ or $E=10$ local batch updates. Each training session is repeated 5 times using different random seeds for weight initialization 


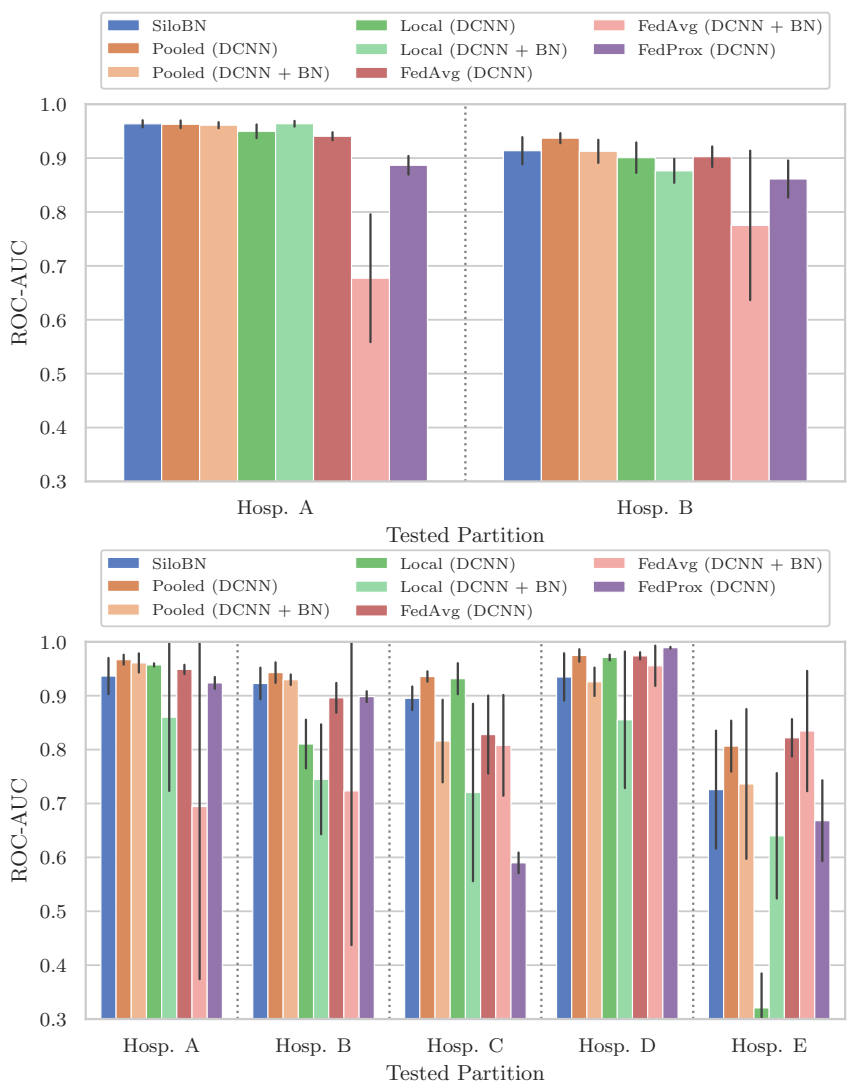

Fig. 2. Predictive performance of different training approaches used for training DCNN with and without BN layers. Performance is measured on per-center held-out test sets. Note that the Pooled, FedProx, and FedAvg approaches produce a single model which is evaluated on multiple test sets, while the Local and SiloBN approaches produce one model per center, which is then evaluated on its corresponding center-specific test set.

and data ordering. Finally, all local optimization is performed using Adam [15]. Tab. 4 in Supp. Mat. provides all related training hyperparameters.

Evaluation To compare the different methods, we measure intra-center generalization performance by computing the area under the curve (AUC) of the ROC curve of the trained model's predictions on each center's held-out data for single-model methods (Pooled, FedAvg, or FedProx), and personalized models are tested on held-out data for their specific training domain (Local and SiloBN). We also report mAUC, which corresponds to the pan-center mean of intra-center generalization AUCs. 
Table 1. Mean AUC over federated centers for different training approaches on FLC16 and FL-C17. The proposed SiloBN is on par or better than other FL methods, and is shown to be the most effective approach when using BN layers.

\begin{tabular}{lccc}
\multicolumn{4}{l}{ WITH BN LAYERS } \\
\hline \multirow{3}{*}{ Pooled } & $E$ & FL-C16 & FL-C17 \\
\cline { 2 - 4 } Local & - & $0.94 \pm 0.03$ & $0.87 \pm 0.11$ \\
SiloBN & - & $0.92 \pm 0.05$ & $0.76 \pm 0.16$ \\
\cline { 2 - 4 } & 1 & $0.94 \pm 0.03$ & $0.86 \pm 0.13$ \\
FedAvg & $\mathbf{1 0}$ & $\mathbf{0 . 9 4} \pm \mathbf{0 . 0 3}$ & $\mathbf{0 . 8 8} \pm \mathbf{0 . 1 0}$ \\
& 1 & $0.81 \pm 0.05$ & $0.70 \pm 0.18$ \\
& 10 & $0.73 \pm 0.14$ & $0.80 \pm 0.22$ \\
\hline
\end{tabular}

\begin{tabular}{lccc}
\multicolumn{4}{c}{ Without BN LAYERs } \\
\hline & $E$ & FL-C16 & FL-C17 \\
\cline { 2 - 4 } Pooled & - & $0.95 \pm 0.02$ & $0.93 \pm 0.07$ \\
Local & - & $0.93 \pm 0.03$ & $0.80 \pm 0.25$ \\
\cline { 2 - 4 } & & & \\
FedProx & 10 & $0.87 \pm 0.03$ & $0.81 \pm 0.16$ \\
FedAvg & 1 & $0.62 \pm 0.15$ & $0.80 \pm 0.17$ \\
& $\mathbf{1 0}$ & $\mathbf{0 . 9 2} \pm \mathbf{0 . 0 2}$ & $\mathbf{0 . 8 9} \pm \mathbf{0 . 0 7}$ \\
\hline
\end{tabular}

Table 2. Domain generalization of SiloBN and FedAvg (DCNN) from FL-C16 to FLC17. On average, SiloBN outperforms FedAvg and yields more stable results.

\begin{tabular}{lccccc|c}
\hline Hospital & $\mathrm{A}$ & $\mathrm{B}$ & $\mathrm{C}$ & $\mathrm{D}$ & $\mathrm{E}$ & Mean \\
\cline { 2 - 7 } SiloBN+AdaBN & $0.94 \pm 0.01$ & $\mathbf{0 . 9 1} \pm \mathbf{0 . 0 4}$ & $0.93 \pm 0.01$ & $\mathbf{0 . 9 8} \pm \mathbf{0 . 0 1}$ & $\mathbf{0 . 9 5} \pm \mathbf{0 . 0 1}$ & $\mathbf{0 . 9 4} \pm \mathbf{0 . 0 2}$ \\
FedAvg (DCNN) & $\mathbf{0 . 9 5} \pm \mathbf{0 . 0 2}$ & $0.86 \pm 0.03$ & $\mathbf{0 . 9 5} \pm \mathbf{0 . 0 1}$ & $\mathbf{0 . 9 8} \pm \mathbf{0 . 0 2}$ & $0.88 \pm 0.03$ & $0.92 \pm 0.05$ \\
\hline
\end{tabular}

Impact of Federated Approaches Fig. 2 provides per-center testing performance for $E=10$ updates on FL-C16 and FL-C17 and Tab. 1 shows a full table of results averaged across centers, including a comparison of performance over the number of local steps per federated round. On both datasets, the proposed SiloBN outperforms other FL strategies for the DCNN+BN network, and is on par with the pooled and local trainings. For the DCNN network, for which FedAvg and SiloBN are equivalent, we can make the same observation. However, the lack of BN layers increases the sensitivity of FedAvg to E. We note that for FL-C17, some reported results in Fig. 2 have very large error bars or very low AUC. More analysis is required to understand the source of these instabilities. Since some of these odd results occur for local trainings, they could e.g. stem from poor local data quality. Overall, from this set of experiments, we conclude that the proposed SiloBN is the only method that can fully utilize BN layers in the FL setting.

\subsection{Out-of-domain generalization experiments}

We now investigate the ability of the proposed method to transfer to new domains. We first train a model on FL-C16 and test it on FL-C17. We compare the proposed SiloBN, where testing is done with $A d a B N$ as explained in Sec. 4, to a DCNN without BN layers trained with FedAvg, for which no adaptation is needed. Per-hospital generalization results are provided in Tab. 2, We note that the proposed algorithm outperforms the FedAvg-trained DCNN model on average across centers. Moreover, we note that its generalization performance is much more stable, both across trainings (lower variance) and across centers. 


\section{Conclusion}

We have introduced SiloBN, a novel FL strategy which relies on local-statistic BN layers in DCNNs. Experiments on real-world multicentric histopathology datasets have shown that this method yields similar, or better, intra-center generalization capabilities than existing FL methods. Importantly, the proposed approach outperforms existing methods on out-of-domain generalization in terms of performance and stability, while enjoying a better privacy profile by not sharing local activation statistics. Future works could quantify the privacy benefits of this approach as well as study its applicability to other domains, e.g. radiology.

\section{References}

1. Abadi, M., Chu, A., Goodfellow, I., McMahan, H.B., Mironov, I., Talwar, K., Zhang, L.: Deep learning with differential privacy. In: Proc. ACM Conf. on Comp. Comm. Sec. (Oct 2016)

2. Bandi, P., et al.: From detection of individual metastases to classification of lymph node status at the patient level: the camelyon 17 challenge. IEEE transactions on medical imaging 38(2), 550-560 (2018)

3. Bejnordi, B.E., et al.: Diagnostic assessment of deep learning algorithms for detection of lymph node metastases in women with breast cancer. Jama $\mathbf{3 1 8}(22)$, 2199-2210 (2017)

4. Bonawitz, K., Ivanov, V., Kreuter, B., Marcedone, A., McMahan, H.B., Patel, S., Ramage, D., Segal, A., Seth, K.: Practical secure aggregation for privacy-preserving machine learning (2017), iACR Cryptology Preprint

5. Bonawitz, K., et al.: In: Proc. SysML Conference. Palo Alto, CA (2019)

6. Caldas, S., Wu, P., Li, T., Konečnỳ, J., McMahan, H.B., Smith, V., Talwalkar, A.: Leaf: A benchmark for federated settings. arXiv preprint arXiv:1812.01097 (2018)

7. Cireşan, D.C., Giusti, A., Gambardella, L.M., Schmidhuber, J.: Mitosis detection in breast cancer histology images with deep neural networks. In: International Conference on Medical Image Computing and Computer-assisted Intervention. pp. 411-418. Springer (2013)

8. Courtiol, P., et al.: Deep learning-based classification of mesothelioma improves prediction of patient outcome. Nature medicine 25(10), 1519-1525 (2019)

9. Dimitriou, N., Arandjelović, O., Caie, P.D.: Deep learning for whole slide image analysis: An overview. Frontiers in Medicine 6 (2019)

10. Goetz, J., Malik, K., Bui, D., Moon, S., Liu, H., Kumar, A.: Active federated learning (2019), arXiv Preprint [cs.LG]:1909.12641

11. He, K., Zhang, X., Ren, S., Sun, J.: Deep residual learning for image recognition. In: The IEEE Conference on Computer Vision and Pattern Recognition (CVPR). pp. 770-778 (June 2016)

12. Hu, J., Shen, L., Sun, G.: Squeeze-and-excitation networks. In: Proceedings of the IEEE Conference on Computer Vision and Pattern Recognition. pp. 7132-7141 (2018)

13. Ioffe, S., Szegedy, C.: Batch normalization: Accelerating deep network training by reducing internal covariate shift. arXiv preprint arXiv:1502.03167 (2015)

14. Kairouz, P., et al.: Advances and open problems in federated learning (2019), arXiv Preprint [cs.LG]:1912.04977 
15. Kingma, D., Ba, J.: Adam: A method for stochastic optimization (December 2014), arXiv Preprint [cs.LG]:1412.698

16. Komura, D., Ishikawa, S.: Machine learning methods for histopathological image analysis 16, 34-42 (2018)

17. Konecný, J., McMahan, H.B., Ramage, D., Richtárik, P.: Federated optimization: Distributed machine learning for on-device intelligence (October 2016), arXiv Preprint [cs.LG]:1610.02527

18. Li, T., Sahhu, A.K., Talwalkar, A., Smith, V.: Federated learning: Challenges, methods, and future directions (2019), arXiv Preprint [cs.LG]:1908.07873

19. Li, X., Huang, K., Yang, W., Wang, S., Zhang, Z.: On the convergence of fedavg on non-iid data (2019), arXiv Preprint [stat.ML]:1907.02189

20. Li, Y., Wang, N., Shi, J., Liu, J., Hou, X.: Revisiting batch normalization for practical domain adaptation. arXiv preprint arXiv:1603.04779 (2016)

21. Litjens, G., et al.: 1399 H\&E-stained sentinel lymph node sections of breast cancer pateints: The CAMELYON dataset. GigaScience $\mathbf{7}(6)$, giy065 (2018)

22. McMahan, H.B., Moore, E., Ramage, D., Hampson, S., y Arcas, B.A.: Communication-efficient learning of deep networks from decentralized data (February 2017), arXiv Preprint [cs.LG]:1602.05629

23. Ronneberger, O., Fischer, P., Brox, T.: U-net: Convolutional networks for biomedical image segmentation. In: International Conference on Medical image computing and computer-assisted intervention. pp. 234-241. Springer (2015)

24. Sahu, A.K., Li, T., Sanjabi, M., Zaheer, M., Talwalkar, A., Smith, V.: On the convergence of federated optimization in heterogeneous networks (2018), arXiv Preprint [cs.LG]: 1812.06127

25. Sattler, F., Mŭller, K.R., Samek, W.: Clustered federated learnig: Model-agnostic distributed multi-task optimization under privacy constraints (2019), arXiv Preprint [cs.LG]:1910.01991

26. Shokri, R., Shmatikov, V.: Privacy-preserving deep learning. In: Proceedings of the 22nd ACM SIGSAC conference on computer and communications security. pp. 1310-1321. ACM (2015)

27. Shokri, R., Stronati, M., Song, C., Shmatikov, V.: Membership inference attacks against machine learning models. In: 2017 IEEE Symposium on Security and Privacy (SP). pp. 3-18. IEEE (2017)

28. Tan, M., Le, Q.: EfficientNet: Rethinking model scaling for convolutional neural networks. In: Proceedings of the 36th International Conference on Machine Learning. Proceedings of Machine Learning Research, vol. 97, pp. 6105-6114. PMLR, Long Beach, California, USA (09-15 Jun 2019), http://proceedings.mlr.press/ v97/tan19a.html

29. Vapnik, V.: Principles of risk minimization for learning theory. In: Advances in neural information processing systems. pp. 831-838 (1992)

30. Vepakomma, P., Gupta, O., Dubey, A., Raskar, R.: Reducing leakage in distributed deep learning for sensitive health data. AI for Social Good ICLR Workshop (05 2019)

31. Zhang, S., Choromanska, A.E., LeCun, Y.: Deep learning with elastic averaging SGD. In: Advances in Neural Information Processing Systems. pp. 685-693 (2015)

32. Zhu, L., Liu, Z., Han, S.: Deep leakage from gradients. In: Adv. Neural Info. Proc. Sys. (Dec 2019) 


\section{A Supplementary Material}

\begin{tabular}{|c|c|c|c|c|c|c|c|c|c|}
\hline \multicolumn{10}{|c|}{ Training sets } \\
\hline & \multirow{2}{*}{\multicolumn{2}{|c|}{$\begin{array}{c}\text { FL-C16 } \\
\text { B }\end{array}$}} & \multirow[b]{2}{*}{ Sum } & \multicolumn{6}{|c|}{ FL-C17 } \\
\hline Hospital & & & & A & B & $\mathrm{C}$ & $\mathrm{D}$ & $\mathrm{E}$ & Sum \\
\hline Healthy & 50,892 & 40,000 & 90,892 & 4,562 & 3,500 & 4,900 & 3,900 & 3,700 & 20,562 \\
\hline Tumor & 27,078 & 22,981 & 50,059 & 1,740 & 597 & 3,046 & 2,895 & 8,856 & 17,134 \\
\hline Sum & 77,970 & 62,981 & 140,951 & 6,302 & 4,097 & 7,946 & 6,795 & 12,556 & 37,696 \\
\hline \multicolumn{10}{|c|}{ Validation sets } \\
\hline & \multicolumn{3}{|c|}{ FL-C16 } & \multicolumn{6}{|c|}{ FL-C17 } \\
\hline Hospital & A & B & Sum & A & B & $\mathrm{C}$ & $\mathrm{D}$ & $\mathrm{E}$ & Sum \\
\hline Healthy & 9,000 & 21,565 & 30,565 & 1,000 & 1,200 & 1,500 & 1,100 & 1,500 & 6,300 \\
\hline Tumor & 6,292 & 7,284 & 13,576 & 205 & 125 & 259 & 38 & 98 & 725 \\
\hline Sum & 15,292 & 28,849 & 44,141 & 1,205 & 1,325 & 1,759 & 1,138 & 1,598 & 7,025 \\
\hline \multicolumn{10}{|c|}{$\overline{\text { Test sets }}$} \\
\hline & \multicolumn{3}{|c|}{ FL-C16 } & \multicolumn{6}{|c|}{ FL-C17 } \\
\hline Hospital & A & B & Sum & A & B & $\mathrm{C}$ & $\mathrm{D}$ & $\mathrm{E}$ & Sum \\
\hline Healthy & 20,362 & 10,000 & 30,362 & 800 & 1,100 & 1,100 & 1,000 & 900 & 4,900 \\
\hline Tumor & 6,115 & 9,159 & 15,274 & 523 & 847 & 647 & 1,088 & 912 & 4,017 \\
\hline Sum & 26,477 & 19,159 & 45,636 & 1,323 & 1,947 & 1,747 & 2,088 & 1,812 & 8,917 \\
\hline
\end{tabular}

Table 3. Distribution of healthy and tumor-containing tile images across centers for each of the constructed federated datasets.

\begin{tabular}{|c|c|c|c|c|c|c|}
\hline Network & Method & $\beta_{1}$ & $\beta_{2}$ & $\begin{array}{c}\text { L2 } \\
\text { reg. }\end{array}$ & $\begin{array}{c}\text { FedProx } \\
\text { reg. }\end{array}$ & $\begin{array}{c}\text { Learning } \\
\text { rate }\end{array}$ \\
\hline DCNN + BN & $\begin{array}{c}\text { All except FedAvg } \\
\text { DCNN + BN }\end{array}$ & 0 & 0.99 & 0.001 & - & $10^{-4}$ \\
FedAvg & 0 & 0.999 & 0.1 & - & $10^{-3}$ \\
\hline DCNN & All except Fedprox & 0 & 0.99 & 0 & - & $10^{-3}$ \\
DCNN & FedProx & 0 & 0.99 & 0 & 0.1 & $10^{-3}$ \\
\hline \hline Dataset & Network & Method & E & Batch size & Number of rounds \\
\hline FL-C16 & All unless specified & - & 1 & 32 & 21875 \\
FL-C16 & All unless specified & - & 10 & 32 & 2187 \\
FL-C16 & DCNN+BN & Pooled & - & 64 & 21875 \\
FL-C16 & DCNN & FedAvg & 1 & 32 & 5000 \\
FL-C16 & DCNN & FedProx & 10 & 32 & 300 \\
\hline FL-C17 & All unless specified & - & 1 & 32 & 2355 \\
FL-C17 & All unless specified & - & 10 & 32 & 235 \\
FL-C17 & DCNN+BN & Pooled & - & 160 & 2355 \\
FL-C17 & DCNN & FedAvg & 1 & 32 & 500 \\
FL-C17 & DCNN & FedProx & 10 & 32 & 50 \\
\hline
\end{tabular}

Table 4. Hyperparameters used in our experiments obtained with the validation set. When applicable, the momentum of the BN layers is set to 0.1 and the value eps to $10^{-5}$. The first and second moment decay hyperparameters of the Adam optimizer are noted $\beta_{1}$ and $\beta_{2}$. For each dataset, local training used parameters corresponding to $E=1$. 


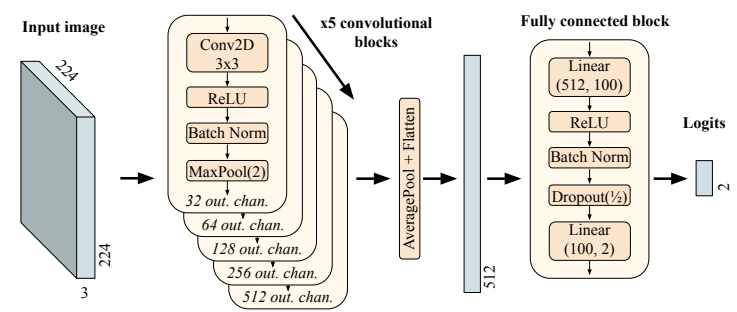

Fig. 3. Architecture of the deep convolutional neural network used in all the experiments. All convolutional layers use zero-padding of size 1 on spatial dimensions. The number of output channels of each convolutional layer corresponds to the number of output channels (abbreviated as out. chan.) of the corresponding convolutional block. All convolutional and linear layers include a bias.

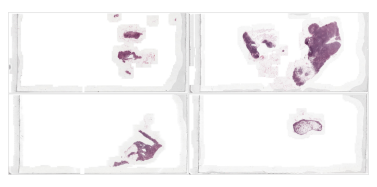

C16: Hosp. A

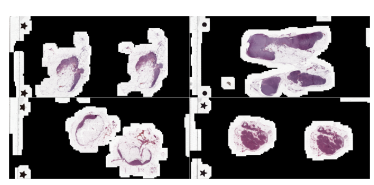

C17: Hosp. B

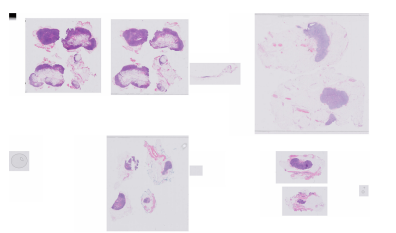

C17: Hosp. E

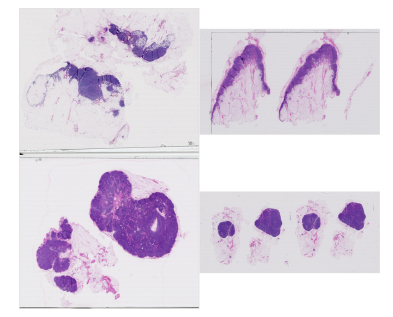

C16: Hosp. B

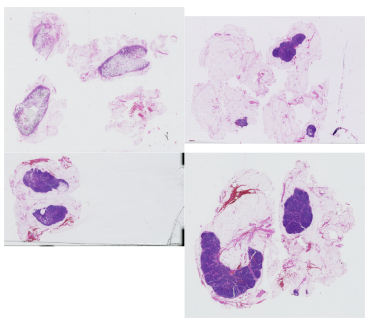

C17: Hosp. C

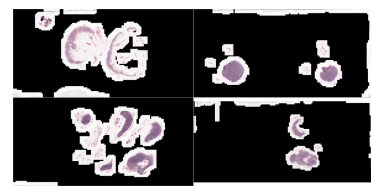

C17: Hosp. A

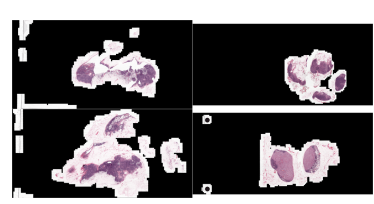

C17: Hosp. D

Fig. 4. Four representative WSIs for each identified center in the Camelyon 16 and Camelyon 17 datasets. 